\title{
Influence of Spatial Statistics of Spectrum Use on the Performance of Cognitive Wireless Networks
}

\author{
Janne Riihijärvi, Jad Nasreddine and Petri Mähönen \\ Institute for Networked Systems, RWTH Aachen University \\ Kackertstrasse 9, D-52072 Aachen, Germany \\ email: \{jar, jad, pma\}@inets.rwth-aachen.de
}

\begin{abstract}
We study the performance characteristics of cognitive wireless networks under different dynamic spectrum access scenarios. Our focus is especially on the influence of spatial structures of the primary and secondary user networks on the achievable performance of the secondary network. We adopt techniques from spatial statistics to develop stochastic models for the structure and interaction of these networks. The chosen models are based on Gaussian random fields and Gibbs point processes, and are firmly grounded on empirical data. We then apply extensive Monte Carlo simulations to study the behavior of these models and their related performance properties. The models and the applied techniques are applicable also to a wider variety of networking problems. Our results provide first quantitative assessments on the influence of the spatial structure, and especially correlation properties, of the involved networks on the expected performance and thus on the utility of dynamic spectrum access based systems.
\end{abstract}

\section{Categories and Subject Descriptors}

C.2 [Computer-Communication Networks]: Miscellaneous; I.6.4 [Simulation and Modeling]: Model Validation and Analysis; I.6.5 [Simulation and Modeling]: Model Development

\section{Keywords}

Performance evaluation, dynamics spectrum access, cognitive wireless networks, spatial statistics

\section{INTRODUCTION}

Cognitive Wireless Networks (CWNs) and in particular Dynamic Spectrum Access (DSA) based systems have become one of the most intensely studied research topics in wireless communications [1-3]. There has been significant progress during recent years in understanding theoretical foundations of the individual technologies involved, as well

Permission to make digital or hard copies of all or part of this work for personal or classroom use is granted without fee provided that copies are not made or distributed for profit or commercial advantage and that copies bear this notice and the full citation on the first page. To copy otherwise, to republish, to post on servers or to redistribute to lists, requires prior specific permission and/or a fee.

MSWiM'12, October 21-25, 2012, Paphos, Cyprus.

Copyright 2012 ACM 978-1-4503-1628-6/12/10 ...\$15.00. as studying different application scenarios for DSA (see, for example, [4-7]). However, the system-level performance properties of dynamic spectrum access networks remain relatively poorly understood, especially when the primary user is not a TV broadcasting network. This is in part due to the very complicated dynamics over space, time, and frequency domains inherent in such networks. These dynamics arise because the reuse of spectrum effectively couples the dynamics of the involved networks together in a manner that does not occur in more traditional wireless communication systems. From practical point of view, most significant results have probably been estimates on available spectrum for DSA use in the context of TV white spaces based on actual TV tower data sets [8-12]. These data sets have also been used to study the potential performance of Wi$\mathrm{Fi}$ or cellular network deployments in the TV bands (see, for example, [13]). Some more general asymptotic capacity results have also been presented in the literature, but these are usually derived assuming either very large-scale multihop networks, or uniformly random deployment of nodes in both primary and secondary networks [14-17].

In this paper we provide detailed results on the influence of spatial structure of the primary and secondary networks on the achievable performance of the secondary system. We formulate a stochastic model for the structure and interaction of the primary and secondary user networks using techniques from spatial statistics [18] in an effort to strike a balance between mathematical tractability and realism of the model. In particular, we argue based on extensive spectrum use measurements and several empirical data sets on wireless network structure that a combination of Gaussian random fields [19] and Gibbs point processes [20] with careful selection of parameters yield good models. While our focus here is on the influence of the spatial structure of the networks involved on performance, the models can be extended towards the full space-time case as well in the manner of $[21]^{1}$. We believe that the modeling approach adopted is also of independent interest, and can be used as a foundation for treatment of wide variety of other types of networking problems, wireless or otherwise. The price to be paid for the added realism in this approach is the scarcity of closed-form analytical results to rely on, except in various asymptotic cases. Accordingly, in order to obtain results that are relevant for expected deployments, we focus instead on the use

\footnotetext{
${ }^{1}$ In this paper we generalize the approach used in [21], where we applied actual cellular network base station locations as a model of the primary network. In the present work much more general spatial models are used.
} 
of Monte Carlo simulations in deriving our performance estimates.

The rest of this paper is structured as follows. In Section II we give a short overview of the DSA scenarios considered in the rest of the paper, and discuss the different design alternatives. We then give the precise model formulation of the arising networks based on the scenarios in Section III. In Section IV we present our results on the arising secondary network structures and their allowed transmit powers, and then extend these results towards capacity estimates in Section V. We finally draw our conclusions in Section VI.

\section{DEFINITION OF THE CONSIDERED SCENARIOS}

Before presenting the modeling framework in detail, we shall briefly recall some basic facts about DSA based networks. The defining property of such networks is that a secondary network can access opportunistically the licensed spectrum of a primary network that is willing to share its spectrum under some constraints. In the classical DSA scenario the primary network is an incumbent holding a license for a range of frequencies used, e.g., for broadcasting services, whereas the secondary user is a packet radio network seeking to use the same range of frequencies when and where the secondary activity does not degrade the primary quality of service more than given by a specified threshold. However, the range of potential applications for DSA techniques is much broader. For example, the primary and secondary systems might be operated by the same operator (e.g. the primary being a mobile network and the secondary an IEEE 802.11 hotspot deployed by the same operator). The secondary network could even be an integrated part of the primary network such as in the case of femtocell network that might need to use some of the allocated sub-channels to the macrocells due to high traffic loads. In our model we do not make specific assumptions about the technologies involved. In particular, primary networks will not be limited to broadcasting networks with very large coverage as is usually considered in the literature (e.g. IEEE 802.22).

The scenario in which DSA is being used defines the constraints the secondary must follow with respect to the access to the primary spectrum. These constraints can be either related to a reduction in the coverage area or to an acceptable interference probability $[6,17,22,23]$. In this paper, we mainly use the interference probability as the primary constraint. However, it should be noted that a mapping between the two approaches can be derived when specific primary networks with known system characteristics are considered. By using the interference probability constraint, the primary network will ensure that all its users have some required level of Quality of Service (QoS), meaning that the outage probability is kept at a low level. This is reflected by the fact that the interference probability experienced by any primary network client should not exceed a threshold $\varepsilon$. Depending on the chosen application scenario this can either be achieved by on-line algorithms estimating the interference probability for a given secondary network configuration, or by defining through off-line means a fixed spectrum access policy for secondary use. The latter can be based on, for example, use of an energy detector and a limit on maximum allowed transmit power.

The interference probability with respect to primary user, $p$, is the probability that the experienced interference $I$ by $p$ due to secondary activity exceeds a predefined threshold $\iota_{\max }$

$$
\mathbb{P}_{\text {int }} \equiv \mathbb{P}\left\{I \geq \iota_{\max }\right\}
$$

In general, one can define a spectrum hole as "the region of space-time-frequency in which a particular secondary use is possible" [6]. In this paper we focus on the spatial dimension, defining spectrum holes as regions in which either the secondary can transmit with a positive power while respecting the interference constraint, or in which the given energy detection threshold $\theta_{\mathrm{E}}$ is not exceeded. These will be referred to as the power control model and the energy detection model in the following, respectively.

In order to relate the interference probability to the spatial structure of the involved networks, a model for interference and thus for radio propagation is needed. We assume that the interference $I$ experienced by the primary due to the activity of a secondary user at distance $d$ and transmitting with power $P$ is given in logarithmic scale by

$$
I(\mathrm{dBm})=P(\mathrm{dBm})-L(d)+\xi,
$$

where $L(d)$ is the large-scale deterministic path loss and $\xi$ is a normal random variable used to model shadowing. Unless mentioned otherwise, we assume $\xi$ to have zero mean, and standard deviation of $\sigma_{s}=7 \mathrm{~dB}$. For path loss $L(d)$, we adopt the Xia-Bertoni propagation model [24] defined for a given frequency $f$ (expressed in $\mathrm{GHz}$ ) and distance $d$ by

$$
L(d)=K+A_{1} \log _{10}(f)+A_{2} \log _{10}(d),
$$

where the constants $K, A_{1}$ and $A_{2}$ are taken to have values of $122.1 \mathrm{~dB}, 21 \mathrm{~dB}$ and $37.6 \mathrm{~dB}$, respectively. With these assumptions and considering $\varepsilon$ as the threshold of the interference probability the primary constraint can be written using (1) and (2) as

$$
\frac{1}{2}\left[1-\operatorname{erf}\left(\frac{\iota_{\max }+L(d)-P}{\sigma_{s} \sqrt{2}}\right)\right]=\varepsilon .
$$

Let us now relate the primary constraint (4) to the considered scenarios. In the scenario based on energy detection, the size of the no-talk zone [6], in which the secondary user is not allowed to transmit, depends on the distribution of power received from the primary, and the energy detection threshold $\theta_{E}$. The latter is in turn set by, for example, a regulator or the operator of the primary network based on the maximum allowed transmit power $P$, maximum interference $\iota_{\max }$, interference probability $\varepsilon$ and the propagation model. Further, assumptions on primary and secondary network structure are needed to make the connection to the interference probability from these quantities. A typical example of this scenario is the IEEE 802.22 system $[11,12]$. For further discussion on detector based approaches, see $[5,7,25]$.

In the scenario based on power control, the definition of the spectrum hole becomes more flexible. In this scenario, the no-talk zone is reduced to match the coverage area of the primary ${ }^{2}$ and the allowed transmit power of the secondary user will depends on its relative position towards the primary coverage area, or the nearest client of the primary network within this coverage area [26-28]. Implementing such an approach is, of course, more challenging as the

\footnotetext{
${ }^{2}$ This is due to the fact that the receiver can be at any place inside the coverage area, and therefore no secondary can be allowed to be active there.
} 
coverage area of the primary has to be either communicated explicitly to the secondary (resulting effectively into a geolocation-based scheme) or estimated on-line. The latter can be accomplished using collaborative measurements and techniques from spatial statistics and signal processing communities [29-31]. Once the distance $d$ to the coverage area or the nearest primary client has been estimated, the secondary network can invert (4) to obtain the allowed transmit power, given by

$$
P(\mathrm{dBm})=\iota_{\max }+L(d)+\sigma_{s} \sqrt{2} \operatorname{erf}^{-1}(2 \varepsilon-1) .
$$

\section{MODELS OF NODE LOCATIONS AND SPECTRUM USE}

We shall now introduce the models used for primary and secondary networks that will be used throughout the rest of the paper to study the performance characteristics of the two scenarios introduced above. We shall begin by introducing our model of the primary network, and then consider deployment models for the secondary system.

\subsection{Random Field Models of Primary Spectrum Use}

The influence of the primary network on the secondary one is usually studied by separately modeling the locations of primary transmitters, and combining these with models of transmitter activity and radio propagation to arrive at a value of the power spectral density (PSD) at each point in space. This results in models with large number of parameters that tend to be fixed to particular technologies and deployment scenarios. We instead aim at constructing a more parsimonious family of models, combining all of these effects into a single stochastic model that still can be fitted to empirical data. More precisely, we shall assume that the power spectral density of the primary transmitter network (measured in $\mathrm{dBm})$ is a realization of a Gaussian random field $Z(\boldsymbol{x})$, with $\boldsymbol{x}$ taking values in some chosen domain $D \subset \mathbb{R}^{2}$.

The actual marginal PSD distributions for large number of transmitter usually have heavier upper tails than Gaussian random fields, closer to $\alpha$-stable distributions [32]. However, for our applications the property that matters is the lower tail of the marginal distribution that is usually very well approximated by Gaussian random fields. This assumption is, of course, related to the common practice of approximating sums of certain log-normal random variables by a log-normal random variable [33], and seems to hold well in practice as illustrated in Figure 1. For more details on the measurement campaign based on which the figure was generated, see [34].

Assuming now second-order stationarity and isotropy such a model is defined by the mean $\mu_{Z} \equiv \mathbb{E}\{Z(\boldsymbol{x})\}$ and the covariance function

$$
C(r) \equiv \operatorname{Cov}\left(Z(\boldsymbol{x}), Z\left(\boldsymbol{x}^{\prime}\right)\right),
$$

where $r \equiv\left\|\boldsymbol{x}-\boldsymbol{x}^{\prime}\right\|$. We choose the covariance function considered here based on extensive measurement campaigns we have conducted on spectrum use over space, described in detail in $[34,35]$. Based on those results we shall assume that $C(r)$ is given by the Matérn model, defined by

$$
C_{\text {Matérn }}(r) \equiv \sigma^{2}\left(\frac{1}{2^{\kappa-1} \Gamma(\kappa)}\left(\frac{r}{\phi}\right)^{\kappa} K_{\kappa}\left(\frac{r}{\phi}\right)\right),
$$

where $\kappa>0, \phi>0, \sigma^{2}>0, \Gamma$ is the gamma function, and $K_{\kappa}$ is the modified Bessel function of the second kind.

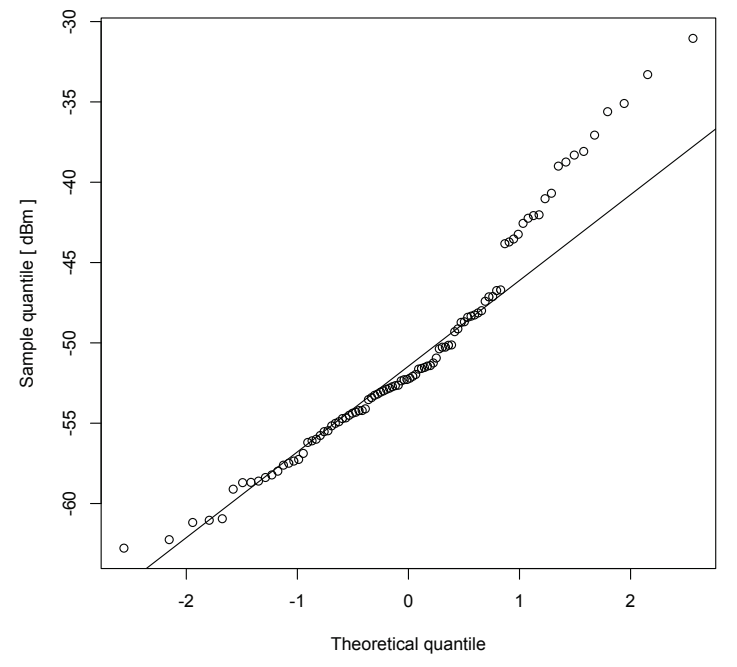

Figure 1: The normal quantile-quantile plot for the marginal PSD distribution for the DVB-T channel 37 measured at 96 locations in downtown Aachen, Germany [34]. The lower tail is clearly very well approximated by the normal distribution.

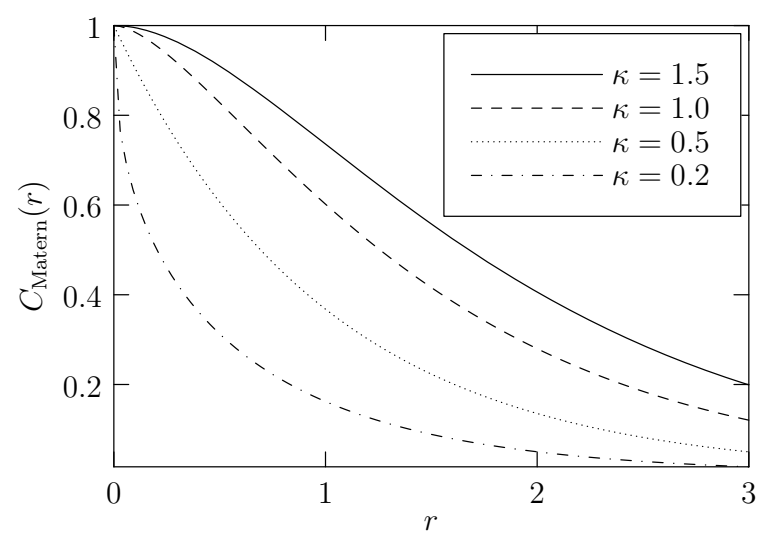

Figure 2: The Matérn covariance function for different values of the smoothness parameter $\kappa$ (with $\left.\sigma^{2}=\phi=1\right)$.

Figure 2 illustrates the shape of the covariance function for different values of $\kappa$. The parameter $\sigma^{2}$ controls the variance of the marginal distribution of $Z$. The effective correlation range of the model is defined as the value of $r$ at which $C(r)=0.05$. It is influenced by both $\phi$ and $\kappa$, with the influence of the latter shown in Figure 3. Typical values of the effective correlation range used below will range from some hundreds of meters to few kilometers. The parameter $\kappa$ is also related to the differentiability of realizations of $Z(\boldsymbol{x})$. Namely, if $\kappa \geq(2 n+1) / 2$ the outcomes of $Z$ will be $n$ times differentiable. Because of this property, we shall refer $\kappa$ as the smoothness of the model.

Corresponding to the two approaches to dynamic spec- 


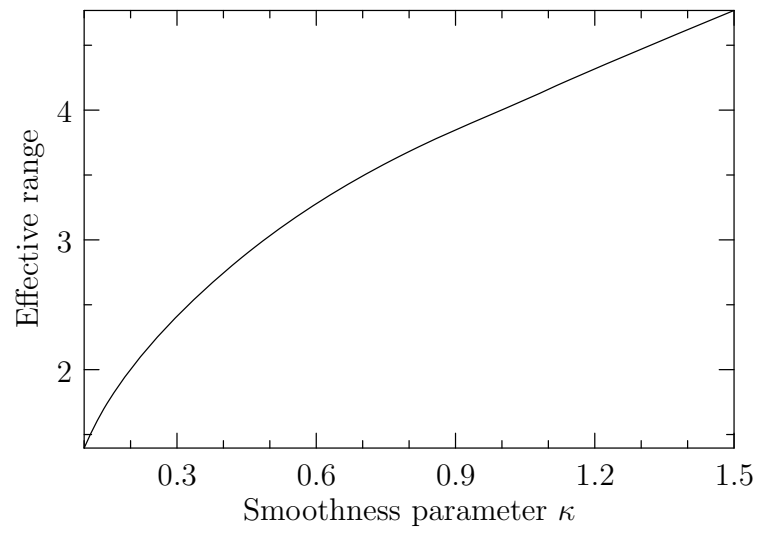

Figure 3: Influence of the smoothness parameter $\kappa$ on the effective range of the Matérn covariance function (with $\sigma^{2}=\phi=1$ ).

trum access discussed in the previous section, we introduce two threshold parameters that together with $Z$ define further random sets that will be central for evaluating the secondary network performance. The energy detection threshold $\theta_{\mathrm{E}}$ defines together with $Z$ the no-talk zone

$$
D_{\mathrm{NT}} \equiv\left\{\boldsymbol{x} \in D \mid Z(\boldsymbol{x}) \geq \theta_{\mathrm{E}}\right\},
$$

inside which secondary use based on energy detection is not allowed. For secondary use based on power control, we define the primary network terminal sensitivity $\theta_{\mathrm{S}}$ yielding the potential primary user service area $^{3}$

$$
D_{\mathrm{SA}} \equiv\left\{\boldsymbol{x} \in D \mid Z(\boldsymbol{x}) \geq \theta_{\mathrm{S}}\right\} .
$$

The service area $D_{\mathrm{SA}}$ defines also another random field, given at each point $\boldsymbol{x} \in D$ as the value of $P$ in (5) evaluated at distance $d=\min _{\boldsymbol{y} \in D_{\mathrm{SA}}}\|\boldsymbol{x}-\boldsymbol{y}\|$. We shall call this random field of allowed transmit powers. Figure 4 shows an example realization of the different random sets and fields defined until now.

\subsection{Models for Node Locations in the Secondary Network}

For modeling the node locations in the secondary network we shall also apply a probabilistic approach. We assume that the locations are given by a point process. Intuitively, a point process is a random process that yields as realizations collections of node locations $\left\{X_{i}\right\}_{i=1}^{n}$. The individual locations $X_{i}$ as well as the total number of locations $n$ are random, but in general with complicated dependency structure. More formally, a point process is defined as a random counting measure $N$, assigning to each measurable set $A \subseteq D$ a random variable $N(A)$, the number of points in $A$ [36]. To make the connection with the more intuitive definition, we can always write

$$
N=\sum_{i=1}^{n} \varepsilon_{X_{i}},
$$

\footnotetext{
${ }^{3}$ Notice that for most actual networks $D_{\text {SA }}$ would be slightly larger than the actual service area, with the difference being dependent on the inter-transmitter interference characteristics of the primary network.
}

where $\varepsilon_{x}$ is a point mass at $x$ (the measure equivalent to Dirac delta distribution), the $X_{i}$ are $D$-valued random variables, and $n$ is a random variable with values in the set of extended natural numbers (i.e., in $\mathbb{N} \cup\{+\infty\}$ ). Usually $N$ is assumed to be simple, meaning that the points $X_{i}$ are almost surely distinct. Due to its analytical tractability, $N$ is often assumed to be a homogeneous Poisson point process in the related work in the literature. We shall instead choose $N$ to yield better correspondence to the spatial structure of actually deployed wireless networks. In earlier work we have shown that the Geyer saturation process [37] provides a very good fit for a number of existing node location data sets [38]. Unfortunately obtaining closed-form analytical solutions within the Geyer model is extremely difficult, and we have to rely on Monte Carlo estimates instead to study the model outcomes. However, we feel this is well justified by the increased realism achieved.

The Geyer saturation model belongs to the family of Gibbs point processes, which are characterized by existence of a density function $f$ with respect to the homogeneous Poisson point process of unit intensity. The Geyer saturation process itself is a generalization of the Strauss process [39], defined by the density

$$
f(\boldsymbol{X})=\alpha \beta^{\#(\boldsymbol{X})} \gamma^{s_{r}(\boldsymbol{X})},
$$

where $s_{r}(\boldsymbol{X})$ denotes the number of point pairs of $\boldsymbol{X}$ that are closer than distance $r$ apart, $\beta>0$ and $0 \leq \gamma \leq 1$. Notice that $\alpha$ is for normalization only, and is not an independent parameter. The Strauss model is powerful tool for modeling regular point processes, in which individual points tend to be separated by some minimum distance, but it cannot model clustered distributions of locations common in wireless networks. For the Geyer process an additional saturation threshold $\zeta$ is added, bounding the contribution of the exponent of $\gamma$ and thereby enabling both clustered (for which $\gamma>1$ ) and regular processes to be modeled. The case $\zeta \rightarrow \infty$ yields the Strauss process as a limit.

The parameters of the Geyer saturation model can be fitted into empirical data sets using maximum likelihood analysis, or pseudolikelihoods. The relevant techniques, including tests for goodness-of-fit, have been primarily developed by Baddeley and Turner, who have also provided an open source implementation of the relevant routines $[40,41]$. We applied these techniques in [38] on, among others, the access point locations of the Google Mountain View Wi-Fi network [42], obtaining an excellent fit with parameters $r=150 \mathrm{~m}, \zeta=2$, $\beta \approx 3.329 \times 10^{-5}$ and $\gamma \approx 0.4112$. The corresponding point process forms our baseline location model, well suited for representing especially deployments of Wi-Fi like dynamic spectrum access networks.

\subsection{Simulation Environment and Parameters}

As discussed above, we shall in several instances rely on Monte Carlo estimates in studying the performance characteristics for the models introduced above. For all the simulations, we consider a square domain $D$ of $10 \mathrm{~km} \times 10 \mathrm{~km}$, resulting in, on average, $N(D)=1350$ secondary user access points being generated. Unless mentioned otherwise, the parameters of the correlation model defining $Z$ are set to $\sigma^{2}=$ $140 \mathrm{dBm}^{2}, \phi=480 \mathrm{~m}, \kappa=1$ and $\mu_{Z}=-90 \mathrm{dBm}$, whereas the thresholds used are set to $\varepsilon=0.05, \theta_{\mathrm{E}}=-105 \mathrm{dBm}$ and $\theta_{\mathrm{S}}=-85 \mathrm{dBm}$ (all given for fixed but arbitrary channel bandwidth). The interference threshold $\iota_{\max }$ of the primary 


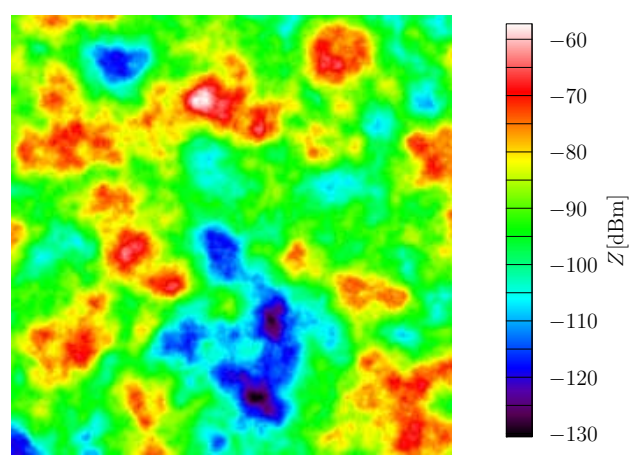

(a) Realization of $Z$ with Matérn covariance.

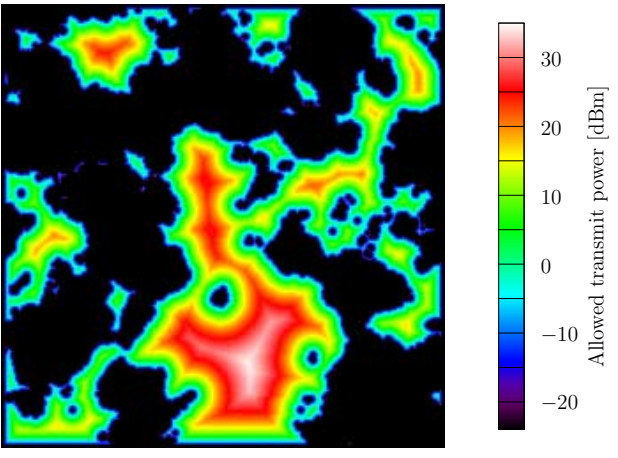

(c) Allowed transmit powers for power control model.

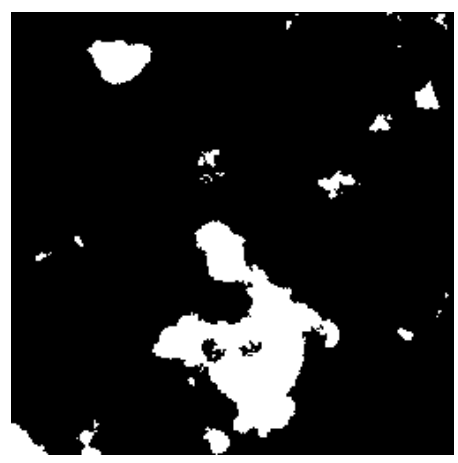

(b) Corresponding random set $D_{\mathrm{NT}}$.

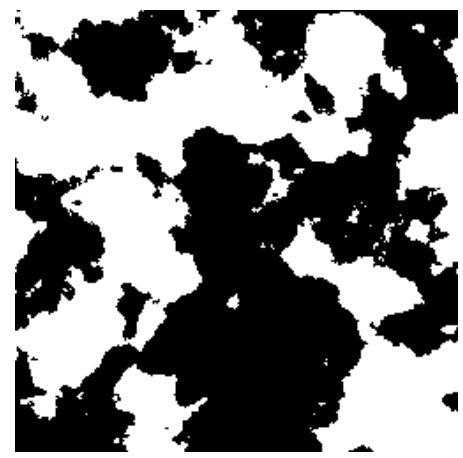

(d) Primary user service area $D_{\mathrm{SA}}$.

Figure 4: Illustrations of the model outcomes with parameters $\kappa=1, \phi=360 \mathrm{~m}, \sigma^{2}=140 \mathrm{dBm}^{2}, \mu_{Z}=-90 \mathrm{dBm}$, $\theta_{\mathrm{E}}=\mathbf{- 1 0 5} \mathrm{dBm}$ and $\theta_{\mathrm{S}}=\mathbf{- 9 0} \mathrm{dBm}$.

and the transmit power for the energy detection model are fixed at $-100 \mathrm{dBm}$ and $20 \mathrm{dBm}$, respectively. The effective correlation range of $Z$ with these parameters is $1920 \mathrm{~m}$. The generated realizations of $Z$ consisted of $250 \times 250$ discrete pixels, each $40 \mathrm{~m} \times 40 \mathrm{~m}$ in size. All the simulations were carried out using the $\mathrm{R}$ environment [43], with the libraries spatstat [41], geoR [44] and RandomFields [45] being used for some of the computations needed. Formal error estimates were used in each case to ensure that simulations were repeated in sufficient numbers, and wherever possible, we report robust statistics of the obtained empirical distributions to ensure maximal confidence in obtained results.

\section{IMPACT OF THE PRIMARY NETWORK STRUCTURE ON SECONDARY TRANSMIT OPPORTUNITIES}

We shall first study the influence of the primary network structure on the occurrence of transmit opportunities for the secondary user based on the models introduced above. We are interested in the number of secondary user nodes that have transmit opportunities in the energy detection and power control models, as well as the quality of those opportunities measured in terms of the allowed transmit power. The related capacity estimates will be discussed in Section V.
The occurrence of transmit opportunities is dependent solely on the structure of the random sets $D_{\mathrm{NT}}$ and $D_{\mathrm{SA}}$. While the shapes of those sets can be complicated, depending on correlation structure of $Z$, their sizes are very simple to characterize. In fact, since the marginal distribution of $Z$ is assumed to be normal, the sizes of $D_{\mathrm{NT}}$ and $D_{\mathrm{SA}}$ and thereby the numbers of nodes of $N$ in these sets depend only on the standardized differences $\Delta_{\mathrm{E}} \equiv\left(\mu_{Z}-\theta_{\mathrm{E}}\right) / \sigma$ and $\Delta_{\mathrm{S}} \equiv\left(\mu_{Z}-\theta_{\mathrm{S}}\right) / \sigma$.

Describing $D_{\mathrm{NT}}$ and $D_{\mathrm{SA}}$ beyond their sizes, and especially their influence on the distribution of active secondary nodes and their allowed transmit powers does not appear feasible through analytic means (although the theory of $e x$ cursion sets [19] would provide some asymptotic results). Accordingly, the following results are obtained from extensive Monte Carlo simulations based on the models introduced above. We shall first consider the energy detection model, and the relation of the spatial structure of active nodes $N_{\mathrm{A}} \equiv N \cap\left(D-D_{\mathrm{NT}}\right)$ to that of our Geyer saturation model $N$.

In the limit of the vanishing effective correlation range (effected by letting $\phi \rightarrow 0$ ) the local structure of the active secondary network would approach that of $N$ subjected to independent thinning, with the retention probability dependent on $\Delta_{\mathrm{E}}$. However, for larger effective correlation ranges 


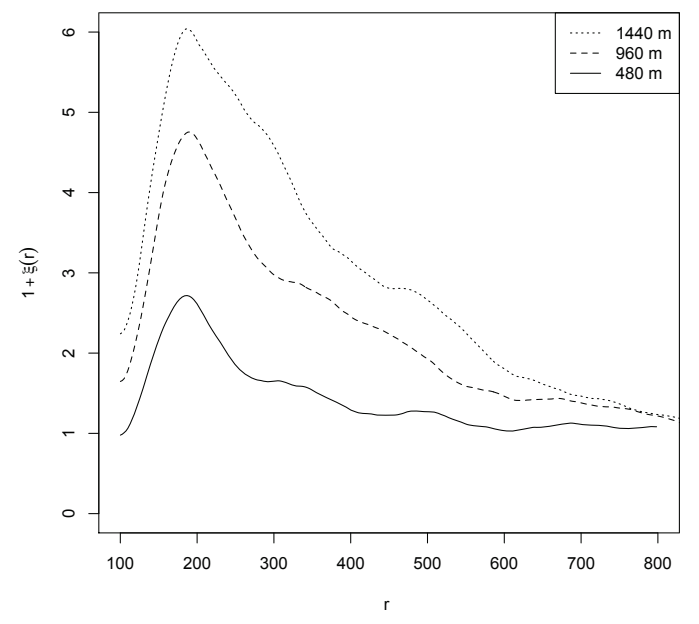

Figure 5: The influence of the effective correlation range of $Z$ on the pair correlation function of active APs in the energy detection model.

the situation is more complex. The correlations in nearby values of $Z$ will cause the set $D-D_{\mathrm{NT}}$ to consist of typically several connected components, with distribution of component sizes being controlled by the correlation function $C(r)$ and the parameter $\Delta_{\mathrm{E}}$. It is intuitively clear that the resulting point process $N_{\mathrm{A}}$ should be clustered. A simple method for confirming this is to estimate the pair correlation function $\xi(r)$ of $N_{\mathrm{A}}$. It is defined by the joint probability density

$$
d P=\nu^{2}(1+\xi(r)) d A_{1} d A_{2}
$$

of finding one point in each of the two area elements $d A_{1}$ and $d A_{2}$ at distance $r$ apart ( $\nu$ is the intensity of the locations, giving the mean number of points per unit area). If the locations were uniformly random, we would obviously have $\xi(r) \equiv 0$. At length scales in which clustering is present, $\xi$ obtains positive values proportional to the level of clustering of locations. Figure 5 shows the estimate of $\xi(r)$ for $N_{\mathrm{A}}$ for three different effective ranges, with the clustering induced by correlations in $Z$ being clearly visible. The high peak at roughly $r=200 \mathrm{~m}$ is induced by the typical distance to the nearest neighboring node in the Geyer model, whereas the high values of $\xi$ around that point are due to the correlations in $Z$.

These correlations are especially significant in scenarios in which $N$ becomes very dense, since they will heavily influence the interference and contention statistics in the secondary network. For a recent interesting asymptotic analysis of this influence, see [46].

In the power control model the structure of the components of $D-D_{\mathrm{SA}}$ influence mainly the distribution of the allowed transmit powers for the active nodes. We shall study both the influence of the sensitivity threshold $\theta_{\mathrm{S}}$ as well as the correlation structure of $Z$ on the results. Notice that since we assume $N$ to be independent of $Z$, the marginal distribution of the random field of allowed transmit powers will also closely reflect the distribution of allowed transmit powers at the active nodes. Thus we shall only focus on the

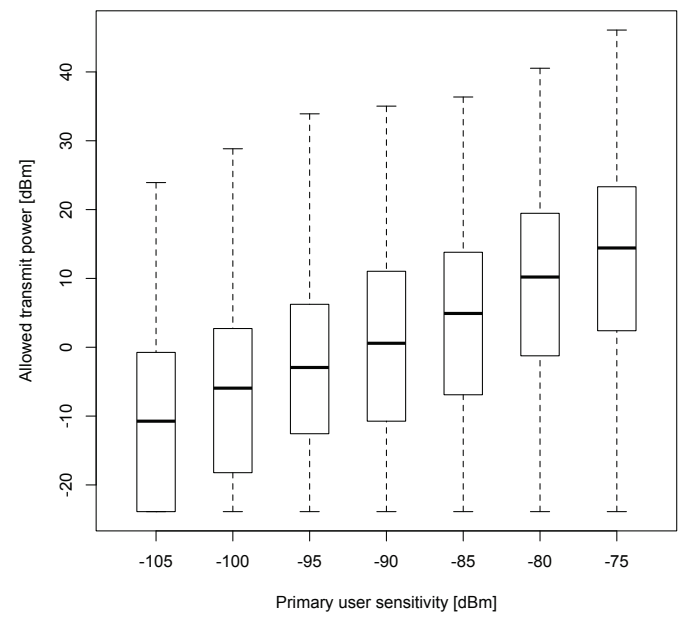

Figure 6: Distribution of the allowed transmit power for the power control model as a function of $\theta_{\mathrm{S}}$. The three horizontal lines of the box correspond to the median and the $25 \%$ and $75 \%$ quantiles of the data, with the whiskers giving the data point at most 1.5 times the interquartile range from the edge of the box.

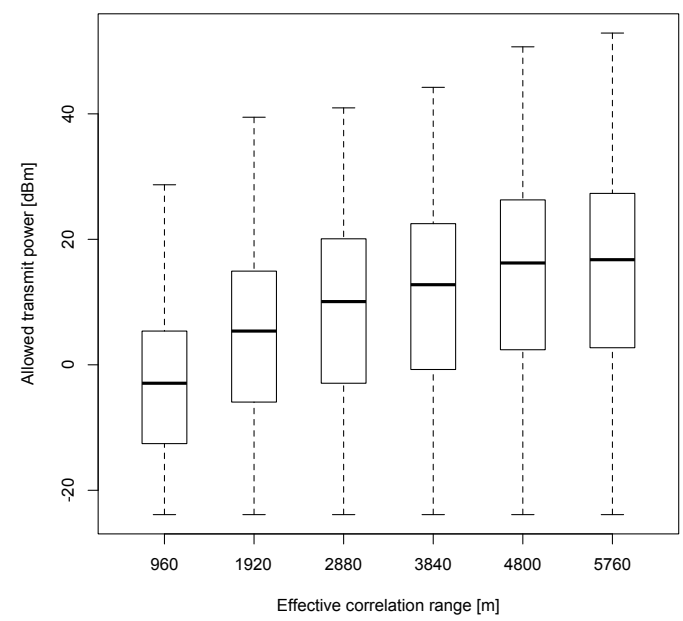

Figure 7: Distribution of the allowed transmit power for the power control model as a function of the effective correlation range of $Z$.

latter in the following. We shall also limit our focus on the distribution of allowed transmit powers of active nodes, as opposed to all the nodes of $N$.

Figure 6 shows the influence of the threshold $\theta_{\mathrm{S}}$ on the distribution of allowed transmit power. The results clearly show that even with the relatively large effective correlation range of $1920 \mathrm{~m}$ threshold values below $-90 \mathrm{dBm}$ will result in very low allowed transmit powers. On the other hand 


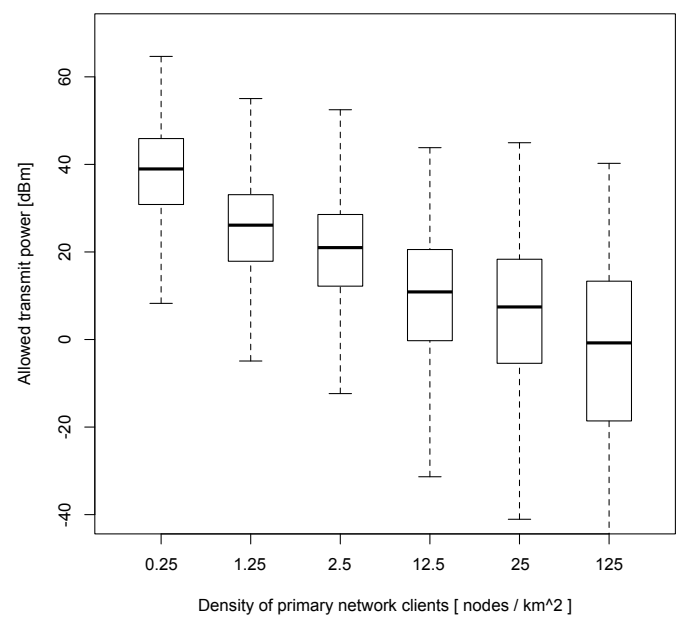

Figure 8: Distribution of the allowed transmit power for the power control model for the case of known primary user receiver positions.

increase in the effective correlation range rapidly increases the quality of transmit opportunities as shown in Figure 7. Especially for effective correlation ranges beyond four kilometers, the active secondary nodes would be able to offer service similar to present-day Wi-Fi hotspots. Of course, the low median powers are in part due to the extremely conservative way of estimating the allowed power. Any further information on expected distribution of the primary user receivers would also significantly increase the resulting transmit powers. A simple illustration of this is shown in Figure 8, corresponding to optimal power control where primary receiver locations are assumed to be known, obtained from a Poisson point process on $D_{\mathrm{SA}}$. Probabilistic models of user distributions could also be used to achieve similar effect, albeit with reduced gains.

We shall conclude this section by studying the influence of the smoothness parameter $\kappa$ on results. Many of the spatial correlation models used in the wireless communications community have assumed a simple parametric correlation model without the possibility to control the smoothness of the arising sample paths. An example of such a model is the exponential one proposed by Gudmundson as a model for correlated shadowing [47], roughly corresponding to the Matérn model with $\kappa=1 / 2$. Because of this, there has been rather few studies on how details, such as the smoothness of the sample paths, influence the estimates obtained using these models. The results for our application, shown in Figure 9 , are quite interesting in this regard. We see that even though the effective correlation range itself is kept constant at $1920 \mathrm{~m}$, small values of $\kappa$ result in very small transmit powers for the secondary network. This is because small values of $\kappa$ allow $Z$ to have occasional high variations in short scales, resulting in small "islands" in $D_{\mathrm{SA}}$ to occur, causing the degradation in allowed transmit powers. The conclusion to be drawn from this is that care should be applied to ensure that for any given application also the sample path behavior of the chosen stochastic model matches that

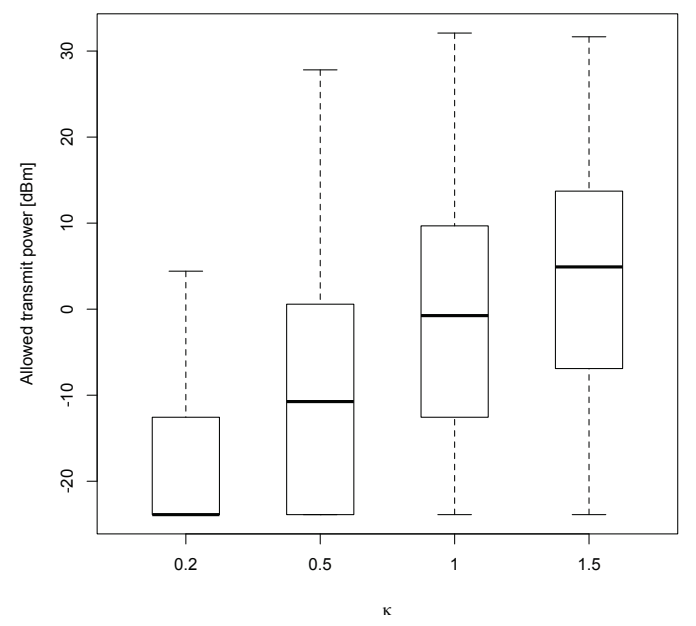

Figure 9: Distribution of the allowed transmit power for the power control model as a function of the smoothness parameter $\kappa$ of $Z$.

of the phenomena being modeled, in addition to correlation range and variance.

\section{ACHIEVABLE CAPACITIES}

We shall now move our focus from allowed transmit powers to capacities. The configuration of the secondary user transmitters can be described in terms of an activation vector $\boldsymbol{a} \in\{0,1\}^{\#(\boldsymbol{X})}$ for which $a_{i}=1$ if the node located at $X_{i}$ active. This in turn depends on $X_{i}$ and the operation of the selected MAC protocol or scheduling solution. We then assume that conditional on an activation vector $\boldsymbol{a}$, the capacity $\mathcal{C}_{i j}$ of the link from $X_{i}$ to $X_{j}$ depends on the signal to interference and noise ratio of the link, given by

$$
\operatorname{SINR}_{i j} \equiv \frac{P_{i} / L_{i j}}{P_{N}+Z\left(X_{j}\right)+\sum_{k} a_{k} P_{k} / L_{k j}},
$$

where $P_{i}$ is the transmit power of $X_{i}, L_{i j}$ is the path loss from $X_{i}$ to $X_{j}, P_{N}$ is noise power, and the summation is over all the links except the one between $X_{i}$ and $X_{j}$. Since we are interested in achievable capacity, we adopt the generalized physical model for which the capacity is estimated by Shannon's formula $\mathcal{C}_{i j}=B \log _{2}\left(1+\mathrm{SINR}_{i j}\right)$, where $B$ is the channel bandwidth, and interference is treated as noise. Alternative choices of capacity metrics are, of course, both possible and straightforward to introduce into the general framework used here. We report all capacity results in normalized form (bits per second per Hertz), since for any reasonable choice of $B$ the system never became dominated by noise.

We focus throughout this section on the secondary network downlink. We assume that around each point of $N$ there is a Poisson distributed number of clients, with mean of five clients per node, and their locations are sampled from a symmetric bivariate normal distribution centered at the points of $N$ with standard deviation of $\sigma=25 \mathrm{~m}$. This can be thought of as a variant of the Thomas point process oc- 


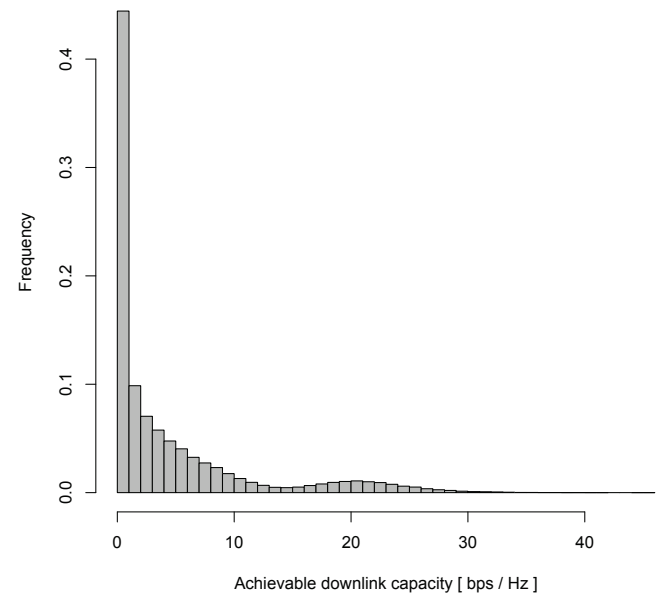

Figure 10: The distribution of achievable downlink capacity for the energy detection model.

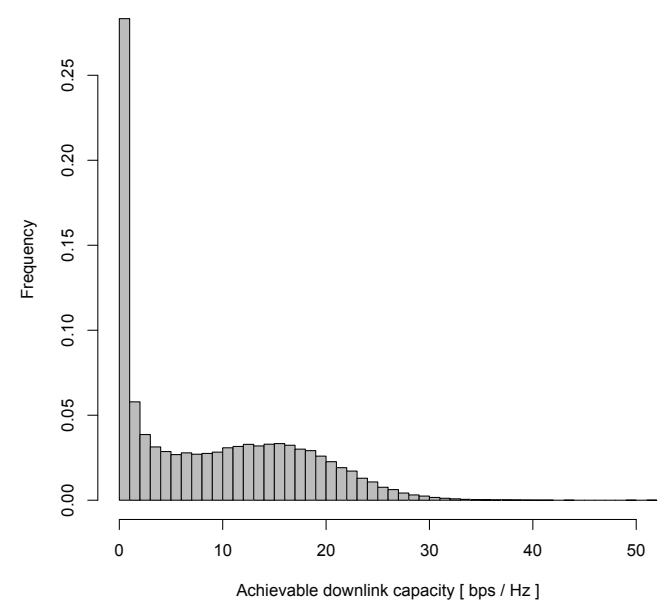

Figure 11: The distribution of achievable downlink capacity for the power control model.

casionally used in performance studies [32], but with more realistic distribution for cluster locations.

Figures 10 and 11 show the distribution of the achievable capacities, taken over the whole client population. We see that as expected, the power control model in general behaves better, resulting in fewer users being in near-outage, and enabling overall decent performance. Since the transmit power of the energy detection model is fixed at relatively high power $(20 \mathrm{dBm})$, some of the clients will have very high SINR-values and correspondingly high capacity. However, the small number of active access points leads into poor overall performance. Figures 12 and 13 show the influence of the effective correlation range of $Z$ on the results. In-

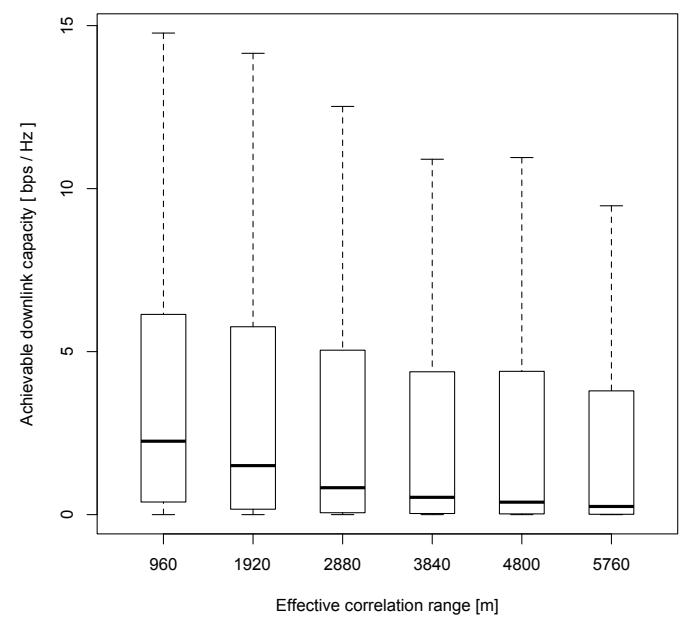

Figure 12: The influence of effective correlation range on achievable downlink capacities for the energy detection model.

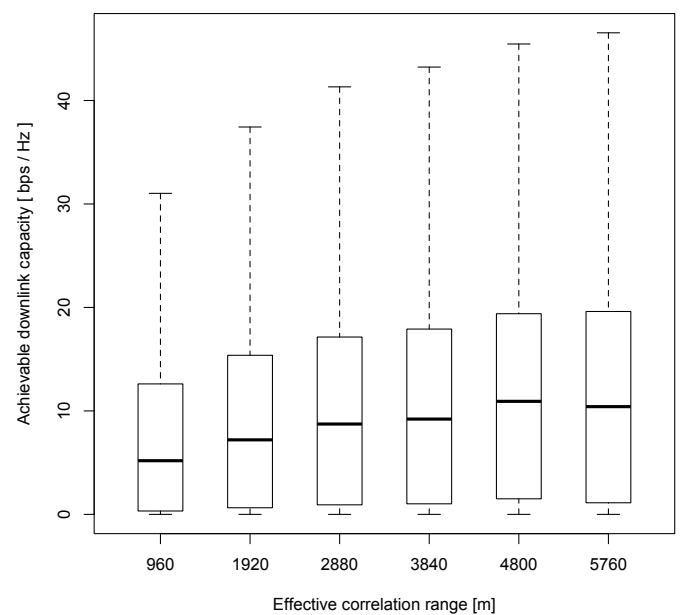

Figure 13: The influence of effective correlation range on achievable downlink capacities for the power control model.

terestingly, increase in correlation range has opposite effects on the downlink capacity distributions for the energy detection and power control models. This is because with the power control model the secondary network can increase link capacities by increasing transmit powers as the correlation length increases (as was shown in Figure 7), and is also able to offer service in larger proportion of the overall domain $D$ (since $\theta_{\mathrm{E}}<\theta_{\mathrm{S}}$ ).

The results discussed above are upper limits, since they assume only one active link in the secondary network. In a more realistic scenario multiple secondary transmitters will be active simultaneously, resulting in increased interference and thereby reduction in capacity. We have also studied 
this case in detail, and while the distribution of capacities does shift lower as more and more nodes become active, the performance did not degrade severely in any of our simulations, and even with all nodes of $N$ transmitting simultaneously the median link capacity was reduced only by approximately $20 \%$ (corresponding figures are omitted for space reasons). This is because of the structure of $N$ which is regular enough to ensure that the inter-transmitter distances are typically quite large. For a denser deployment, or in an ad hoc networking scenario, interference between secondary users would rapidly become an additional performance bottleneck to the limitations imposed by the primary.

\section{CONCLUSIONS}

In this paper we have studied in detail the influence of the spatial structure of the primary network on the performance characteristics of dynamic spectrum access based networks. We have also presented simple yet powerful stochastic modeling framework on which our work was based on. Our experiences indicate that Gaussian random fields and Gibbs point processes fitted to empirical data yield a solid foundation for studying in detail characteristics of DSA networks, and, more generally, cognitive wireless networks. The results of our study show clearly that details of the primary network structure and the level of interference the primary is ready to tolerate have a major impact on the expected secondary performance.

The presented work can of course be extended in several ways. Perhaps the simplest extension would be to consider multiple independent frequency channels instead of a single one. This would greatly reduce the outage probability especially for the energy detection model, and would increase the overall power budget and the resulting capacity of the secondary network. Another worthwhile extension would be to consider more realistic models for the physical and MAC layers. Understanding the upper performance limits is important, but should not be the exclusive goal. The most ambitious extension would finally be to develop a full spacetime version of the presented models, and extend the treatment from one slice of time towards full dynamics of the primary and secondary networks. The major challenge in such a work is, in addition to greatly more complex analysis required, the lack of sufficient empirical data sets on which models could be constructed on. We have taken some early steps towards this direction in [21], albeit using a less general model of the primary network structure as done here.

Finally, we note that the methods applied here have numerous potential applications beyond the cognitive wireless networks. For example, many of the natural inhomogeneities in wireless systems, such as uneven distributions of users, correlated shadowing, or changes in parameters of the propagation environment are amenable to be treated as correlated random fields. Similarly, whenever models of user or node locations are needed that can be fitted to empirical location data, Gibbs point processes form a very powerful tool.

\section{Acknowledgment}

The authors would like to thank RWTH Aachen University and the German Research Foundation (Deutsche Forschungsgemeinschaft, DFG) for providing financial support through the UMIC research centre. We would also like to thank the
European Union for providing partial funding of this work through the FARAMIR project.

\section{REFERENCES}

[1] J. Mitola III and G. Maguire Jr, "Cognitive radio: making software radios more personal," IEEE personal communications, vol. 6, no. 4, pp. 13-18, 1999.

[2] Q. Zhao and B. Sadler, "A survey of dynamic spectrum access," IEEE Signal Processing Magazine, vol. 24, no. 3, pp. 79-89, 2007.

[3] S. Haykin, "Cognitive radio: brain-empowered wireless communications," IEEE journal on selected areas in communications, vol. 23, no. 2, pp. 201-220, 2005.

[4] R. Tandra and A. Sahai, "SNR walls for signal detection," IEEE Journal on Selected Topics in Signal Processing, vol. 2, no. 1, pp. 4-17, 2008.

[5] T. Yucek and H. Arslan, "A survey of spectrum sensing algorithms for cognitive radio applications," IEEE Communications Surveys 8 Tutorials, vol. 11, no. 1, pp. 116-130, 2009.

[6] R. Tandra, M. Mishra, and A. Sahai, "What is a spectrum hole and what does it take to recognize one?" Proceedings of the IEEE, vol. 97, no. 5, pp. 824-848, 2009.

[7] A. Ghasemi and E. S. Sousa, "Spectrum sensing in cognitive radio networks: the cooperation-processing tradeoff," Wireless Communications and Mobile Computing, vol. 7, no. 9, pp. 1049-1060, 2007.

[8] A. Sahai, S. Mishra, R. Tandra, and K. Woyach, "Cognitive radios for spectrum sharing," IEEE Signal Processing Magazine, vol. 26, no. 1, pp. 140-145, 2009.

[9] K. Harrison, S. Mishra, and A. Sahai, "How much white-space capacity is there?" in Proc. of IEEE DySPAN 2010, 2010.

[10] J. van de Beek, J. Riihijärvi, A. Achtzehn, and P. Mähönen, "TV White Space in Europe," IEEE Transactions on Mobile Computing, vol. 11, no. 2, pp. 178-188, Feb. 2012.

[11] C. R. Stevenson, G. Chouinard, Z. Lei, W. Hu, S. J. Shellhammer, and W. Caldwell, "IEEE 802.22: The first cognitive radio wireless regional area network standard," IEEE Communications Magazine, vol. 47, no. 1 , pp. $130-138$, january 2009.

[12] C. Cordeiro, K. Challapali, D. Birru, and S. Shankar, "IEEE 802.22: An introduction to the first wireless standard based on cognitive radios," Journal of Communications, vol. 1, no. 1, pp. 38-47, April 2006.

[13] A. Achtzehn, M. Petrova, and P. Mähönen, "On the Performance of Cellular Networks in TVWS," In Proc. of IEEE ICC'12, Ottawa, Canada, 2012.

[14] J. Lee, S. Lim, J. G. Andrews, , and D. Hong, "Achievable transmission capacity of secondary system in cognitive radio networks," IEEE International Conference on Communications, May 2010.

[15] M. Vu, N. Devroye, and V. Tarokh, "An overview of scaling laws in ad hoc and cognitive radio networks," Wireless Personal Communications, vol. 45, no. 3, pp. 343-354, 2008.

[16] S. Srinivasa and S. Jafar, "Cognitive Radio Networks: How Much Spectrum Sharing is Optimal?" in IEEE Global Telecommunications Conference, $200 \%$. GLOBECOM'0\%, 2007. 
[17] E. G. Larsson and M. Skoglund, "Cognitive radio in a frequency-planned environment: some basic limits," IEEE Transactions on Wireless Communications, vol. 7, no. 12, pp. 4800-4806, December 2008.

[18] N. Cressie, Statistics for spatial data. Wiley-Interscience, 1993.

[19] R. Adler and J. Taylor, Random fields and geometry. Springer Verlag, 2007.

[20] J. Møller and R. Waagepetersen, Statistical inference and simulation for spatial point processes. CRC Press, 2004.

[21] J. Riihijärvi, J. Nasreddine, and P. Mähönen, "Influence of primary network structure and dynamics on achievable performance of cognitive wireless networks," in Proc. of IEEE DySPAN 2011. IEEE, 2011.

[22] B. L. Mark and A. O. Nasif, "Estimation of maximum interference-free power level for opportunistic spectrum access," IEEE Transactions on Wireless Communications, vol. 8, no. 5, pp. 2505 -2513, May 2009.

[23] J. Nasreddine, O. Sallent, J. Pérez-Romero, and R. Agustí, "Positioning-based framework for secondary spectrum usage," Physical Communication, vol. 1, no. 2, pp. 121-133, 2008.

[24] L. Maciel, H. Bertoni, and H. Xia, "Unified approach to prediction of propagation over buildings for all ranges of base station antenna height," IEEE Transactions on Vehicular Technology, vol. 42, no. 1, pp. 41-45, 1993.

[25] B. Farhang-Boroujeny and R. Kempter, "Multicarrier communication techniques for spectrum sensing and communication in cognitive radios," IEEE Communications Magazine, vol. 46, no. 4, pp. 80-85, April 2008.

[26] M. Haddad, M. Debbah, and A. M. Hayar, "Distributed power allocation for cognitive radio," in the 9th International Symposium on Signal Processing and Its Applications (ISSPA 2007), Feb. 2007, pp. 1-4.

[27] Y. Yu, H. Murata, K. Yamamoto, and S. Yoshida, "Interference information based power control for cognitive radio with multi-hop cooperative sensing," IEICE Transactions on Communications, vol. E91-B, no. 1 , pp. $70-76,2008$.

[28] J. Nasreddine, J. Riihijärvi, and P. Mähönen, "Location-based adaptive detection threshold for dynamic spectrum access," in Proc. of IEEE DySPAN 2010, Singapore, April 2010.

[29] S. Kim, E. Dall'Anese, and G. Giannakis, "Cooperative Spectrum Sensing for Cognitive Radios Using Kriged Kalman Filtering," IEEE J. Sel. Topics Sig. Proc, 2011, to appear.

[30] J. Riihijärvi, P. Mähönen, M. Wellens, and M. Gordziel, "Characterization and Modelling of Spectrum for Dynamic Spectrum Access with Spatial Statistics and Random Fields," in Proc. of 1st International Workshop on Cognitive Radios and Networks (CRNETS), in conjunction with IEEE PIMRC 2008, Cannes, France, September 2008.

[31] B. Sayrac, S. Ben Jemaa, and E. Moulines, "Interference Cartography for Hierarchical Dynamic
Spectrum Access," in Proc. of IEEE DySPAN 2008, 2008, pp. 1-5.

[32] M. Haenggi, J. G. Andrews, F. Baccelli, O. Dousse, and M. Franceschetti, "Stochastic Geometry and Random Graphs for the Analysis and Design of Wireless Networks," IEEE Journal on Selected Areas in Communications, Sep. 2009.

[33] N. Beaulieu and Q. Xie, "An optimal lognormal approximation to lognormal sum distributions," IEEE Transactions on Vehicular Technology, vol. 53, no. 2, pp. 479-489, 2004.

[34] A. Achtzehn et al., "Improving Coverage Prediction for Primary Multi-Transmitter Networks Operating in the TV Whitespaces," accepted to IEEE SECON'12, Seoul, Korea, 2012.

[35] M. Wellens, J. Riihijärvi, and P. Mähönen, "Spatial statistics and models of spectrum use," Computer Communications, vol. 32, no. 18, pp. 1998-2011, 2009.

[36] A. F. Karr, Point Processes and Their Statistical Inference, 2nd ed. Marcel Dekker, 1991.

[37] C. Geyer, "Likelihood inference for spatial point processes," Stochastic Geometry: Likelihood and Computation, pp. 79-140, 1999.

[38] J. Riihijärvi and P. Mähönen, "Modeling Spatial Structure of Wireless Communication Networks," in INFOCOM IEEE Conference on Computer Communications Workshops, 2010, 2010, pp. 1-6.

[39] D. Strauss, "A model for clustering," Biometrika, vol. 62, no. 2, p. 467, 1975.

[40] A. Baddeley and R. Turner, "Practical Maximum Pseudolikelihood for Spatial Point Patterns (with Discussion)," Australian $\&$ New Zealand Journal of Statistics, vol. 42, no. 3, pp. 283-322, 2000.

[41] — - "Spatstat: an R package for analyzing spatial point patterns," Journal of Statistical Software, vol. 12, no. 6, pp. 1-42, 2005, ISSN 1548-7660. [Online]. Available: www.jstatsoft.org

[42] J. Robinson, R. Swaminathan, and E. Knightly, "Assessment of urban-scale wireless networks with a small number of measurements," in Proceedings of the 14th ACM international conference on Mobile computing and networking. ACM New York, NY, USA, 2008, pp. 187-198.

[43] R Development Core Team, $R$ : A Language and Environment for Statistical Computing, R Foundation for Statistical Computing, Vienna, Austria, 2007, ISBN 3-900051-07-0. [Online]. Available: http://www.R-project.org

[44] P. J. Ribeiro Jr and P. J. Diggle, "geoR: a package for geostatistical analysis," $R-N E W S$, vol. 1, no. 2, pp. 14-18, June 2001, iSSN 1609-3631. [Online]. Available: http://CRAN.R-project.org/doc/Rnews/

[45] M. Schlather, RandomFields: Simulation and Analysis of Random Fields, 2009, R package version 1.3.41.

[46] R. K. Ganti and J. G. Andrews, "A new method for computing the transmission capacity of non-poisson wireless networks," in Proc. of ISIT, 2010.

[47] M. Gudmundson, "Correlation model for shadow fading in mobile radio systems," Electronics letters, vol. 27 , no. 23 , pp. 2145-2146, 1991. 